\title{
REVIEW ARTICLE N-myristoylation: from cell biology to translational medicine
}

\author{
Meng Yuan ${ }^{1}$, Zi-han Song ${ }^{1}$, Mei-dan Ying ${ }^{1}$, Hong Zhu ${ }^{1}$, Qiao-jun $\mathrm{He}^{1}$, Bo Yang ${ }^{1}$ and Ji Cao ${ }^{1}$
}

Various lipids and lipid metabolites are bound to and modify the proteins in eukaryotic cells, which are known as 'protein lipidation'. There are four major types of the protein lipidation, i.e. myristoylation, palmitoylation, prenylation, and glycosylphosphatidylinositol anchor. $\mathrm{N}$-myristoylation refers to the attachment of 14-carbon fatty acid myristates to the $\mathrm{N}$-terminal glycine of proteins by N-myristoyltransferases (NMT) and affects their physiology such as plasma targeting, subcellular tracking and localization, thereby influencing the function of proteins. With more novel pathogenic $\mathrm{N}$-myristoylated proteins are identified, the $\mathrm{N}$-myristoylation will attract great attentions in various human diseases including infectious diseases, parasitic diseases, and cancers. In this review, we summarize the current understanding of $\mathrm{N}$-myristoylation in physiological processes and discuss the hitherto implication of crosstalk between N-myristoylation and other protein modification. Furthermore, we mention several wellstudied NMT inhibitors mainly in infectious diseases and cancers and generalize the relation of NMT and cancer progression by browsing the clinic database. This review also aims to highlight the further investigation into the dynamic crosstalk of $\mathrm{N}$ myristoylation in physiological processes as well as the potential application of protein $\mathrm{N}$-myristoylation in translational medicine.

Keywords: N-myristoylation; N-myristoyltransferase; infectious diseases; parasitic diseases; cancers; translational medicine Acta Pharmacologica Sinica (2020) 41:1005-1015; https://doi.org/10.1038/s41401-020-0388-4

\section{INTRODUCTION}

Lipids are one of the principal components that structure the cell membrane and provide the barrier required for cells to survive. In addition, various lipids and lipid metabolites are also generated for modifying proteins in eukaryotic cells in a process known as 'protein lipidation'. Generally, there are four major types of protein lipidation: myristoylation, palmitoylation, prenylation, and glycosylphosphatidylinositol (GPI) anchoring. These lipid modifications have been defined by different functional properties that are classified according to the characteristics of lipid attachment, the covalent bond, the specific sequence on the protein and the enzymes involved [1, 2]. These lipidation distinctions impact the charge, hydrophobicity, and other aspects of targeted-protein chemistry, resulting in marked differences in the physiology of the targeted protein, such as its conformation, trafficking, localization, and binding affinity for cofactors. Therefore, due to the deregulated lipid metabolism that occurs, protein lipidation may contribute to various diseases.

This review primarily summarizes the current knowledge of $\mathrm{N}$ myristoylation with updated study results and discusses the strategy of using $\mathrm{N}$-myristoylation in the treatment of diseases.

\section{N-MYRISTOYLATION AND N-MYRISTOYLTRANSFERASE (NMT)}

$\mathrm{N}$-myristoylation consists of the addition of the 14-carbon fatty acid, myristate, to the $\mathrm{N}$-terminal glycine residue of a protein via a covalent amide bond. In rare cases, including those of Ras GTPases and TNF [3, 4], myristic acid is attached to a lysine residue [5] through an amide bound, a process named lysine myristoylation. Previously, it was observed that the myristate attaches to the nascent peptide in the first $10 \mathrm{~min}$ of translation during protein biosynthesis. Therefore, $\mathrm{N}$-myristoylation is considered a cotranslational modification with the most accurate step occurring after the removal of the methionine initiator by methionine aminopeptidase (MetAP) (Fig. 1a, b). Furthermore, it is recognized that Nmyristoylation can also occur posttranslationally on an internal glycine exposed by caspase cleavage during apoptosis (Fig. 1c). For many protein signaling systems, the $\mathrm{N}$-myristoyl moiety represents an essential feature and contributes to numerous effects, including changing protein stability, influencing protein-protein interactions, enhancing subcellular targeting to organelles or the plasma membrane, and so on [6-10].

\section{Two NMT isozymes}

During the multiple enzymatic steps of $\mathrm{N}$-myristoylation, the process of myristate transfer is completed by NMT, which is classified as a member of the GCN5-related N-acetyltransferase (GNAT) superfamily. Some well-studied structural and biochemical investigations of yeast and human NMTs revealed an ordered Bi Bi kinase mechanism (Fig. 1d) that involves a structural transformation of NMT1 during NMT catalysis. In fact, NMT is ubiquitous in eukaryotes while no protozoans possessed it. Typically, lower eukaryotes (e.g., S. cerevisiae and Drosophila) have only one isoform of NMT. However, for some mammalian species, including humans and mice, two isozymes have been identified, referred to as NMT1 and NMT2. These isozymes are encoded by distinct genes but share approximately $77 \%$ peptide sequence identity with unique substrate specificities in the $\mathrm{N}$-terminus, suggesting a distinct physiologic role of each isozyme in mammals. Additionally, each isozyme has a conserved sequence in the catalytic domain of divergent species, implicating an essential role for each gene family throughout evolution.

\footnotetext{
${ }^{1}$ Zhejiang Province Key Laboratory of Anti-Cancer Drug Research, College of Pharmaceutical Sciences, Zhejiang University, Hangzhou 310058, China Correspondence: Ji Cao (caoji88@zju.edu.cn)
} 


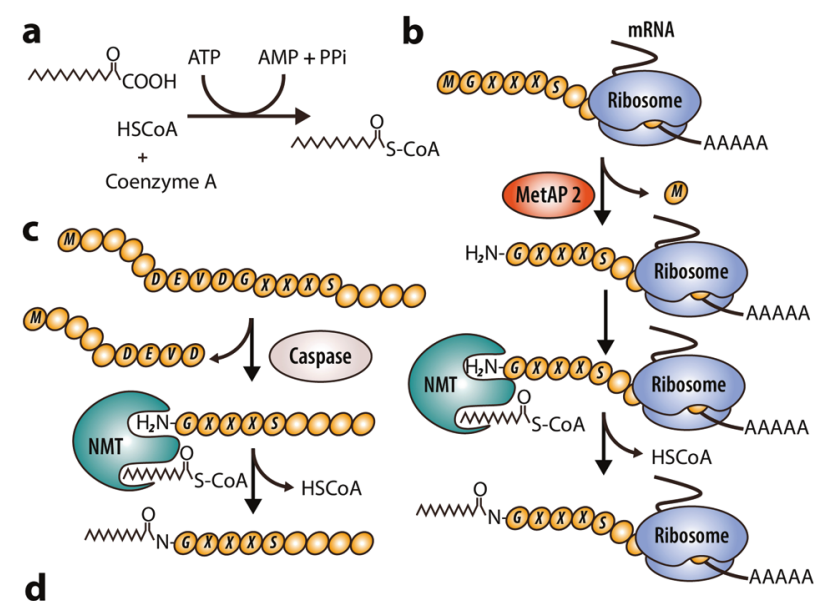

d

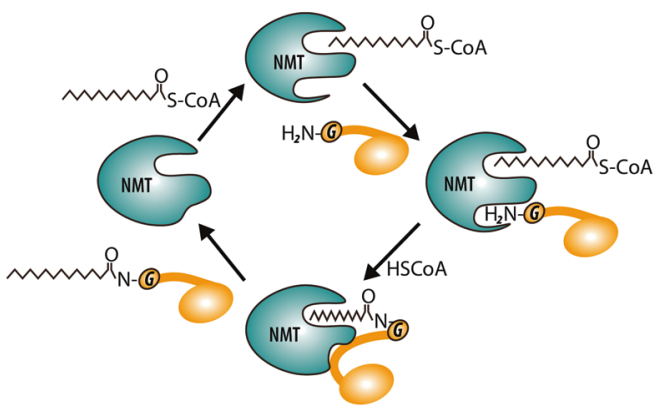

Fig. 1 Schematic pattern of the $\mathrm{N}$-myristoylation mechanism catalyzed by NMTs. a The synthesis of myristoyl-CoA. b Cotranslational modification by $\mathrm{N}$-myristoylation. c Posttranslational modification by $\mathrm{N}$-myristoylation. $\mathbf{d}$ Bi Bi mechanism: The apo-NMT (left) first binds the fatty acid chain of myristoyl-CoA to form the myristoyl-CoA-NMT complex (upper) accompanied by substrate binding pocket exposure. Subsequently, the complex allows a nascent protein to bind (right). Then, the NMT catalyzes $\mathrm{N}$ myristoylpeptide formation through chemical transformation and releases the myristoylpeptide and CoA (lower)

In humans, there are four isoforms of NMT1 and two isoforms of NMT2, which are translated from splice variants of mRNA with different reading frames. The main differences among the NMT isoforms are found in the $\mathrm{N}$-termini. Although the $\mathrm{N}$-terminal structure of these NMTs does not contribute to the construct of the kinase pocket, some investigations have characterized an $\mathrm{N}$ terminal truncation that increases kinase activity without affecting enzyme stability [11]. In addition, the isoforms of these NMTs may have specific effects on their intracellular localization and substrate selectivity $[12,13]$, suggesting that NMTs are involved in diverse physiological processes.

Physiological roles of NMTs

Unequivocally, NMTs play essential roles in the survival and cell proliferation of diverse species $[14,15]$. Some evidence suggests a principal role of NMT1 in the embryonic development of mice. In normal mice, the expression level of NMT1 is similar to that of NMT2 in a wide variety of tissues, but it is higher than the NMT2 levels in embryos. Knocking out NMT1 severely impaired the differentiation ability of embryonic stem cells. Homozygous NMT1 $1^{-1-}$-knockout mice were not born alive, and induction of NMT2 activity alone was unable to elicit the survival of heterozygous $\mathrm{NMT} 1^{+/-}$mice. In addition, it was reported that the subcellular localization and catalytic activity of both NMT1 and NMT2 were altered during apoptosis. NMT1 was transported to the cytosol from ribosomes and membranes following caspase-8- and caspase-3-mediated NMT1 cleavage, and $40 \%$ of the NMT1 activity was eliminated $8 \mathrm{~h}$ after the induction of apoptosis. However, the relocalization of the cytosolic fraction to the membrane and reduced activity of NMT2 were also found under the same conditions. In addition, the depletion of NMT2 caused a 2.3-fold increase in the apoptosis rate compared to the apoptosis rate upon depletion of NMT1 [16]. This evidence led to the speculation that NMT1 may be responsible for ribosome-based cotranslational N-myristoylation, while NMT2 may be the major contributor to apoptosis-related posttranslational N-myristoylation.

In summary, the widespread and conservative presence of NMT1 in different species emphasizes its importance in basic physiological processes, such as embryonic development, while NMT2 appears only in higher-level organisms, suggesting that it is necessary for sophisticated physiological processes. In the future, in-depth biochemical and pharmacological research is expected to improve the understanding of the unique roles of NMT1 and NMT2 as they apply to the clinic.

Protein demyristoylation

Some reports have revealed the demyristoylation ability of some proteins. Human sirtuin 2 (SIRT2), which is a member of the lysine deacetylase sirtuin protein family, was found to exhibit more efficient demyristoylase activity than deacetylase activity [17]. The crystal structure implied that, in complex with a thiomyristoyl peptide, SIRT2 has a dominant hydrophobic pocket that can adopt a myristoyl group. The hydrophobic acyl pocket of SIRT2 resembles that of SIRT6, which has been previously demonstrated to possess efficient fatty acid deacylase activity [18]. In addition, the two pockets are different in certain aspects, suggesting differential adoption of fatty acid chains. Moreover, the other homologs, SIRT1 and SIRT3, each have a hydrophobic acyl pocket very similar to that of SIRT2, hinting at analogous demyristoylation activities among them.

The shigella virulence factor IpaJ was identified as an irreversible demyristoylase [19] that cleaves the peptide bond between $\mathrm{N}$ myristoylated Gly-2 and Asn-3 in some N-myristoylated proteins such as human ADP-ribosylation factor $1 p$ (ARF1) and c-Src. This irreversible demyristoylation mechanism provides a new approach to exploring the functional effects of $\mathrm{N}$-myristoylated proteins in human health and diseases.

\section{CROSS TALK AMONG THE PHYSIOLOGICAL FUNCTIONAL COMPONENTS OF N-MYRISTOYLATION}

In most cases, N-myristoylation on a protein is irreversible, indicating that the myristoyl motif may orient the protein toward a specific destiny, as if it is pressing a button that will irrevocably affect the dynamics of the protein and its subsequent pathway. Therefore, it is reasonable to study the interactions among the factors of $\mathrm{N}$-myristoylation and those of biological signaling pathways to understand the significant role of $\mathrm{N}$-myristoylation. Although N-myristoylation is irreversible, it cannot shield the myristoylated protein from cross talk. In contrast, cross talk is regarded as a means of interfering with $\mathrm{N}$-myristoylation functions. It has been proposed that one protein modification might initiate the signaling that leads to the addition or removal of a second protein modification or the binding of another protein, suggesting that cross talk between protein modification components may serve as an important bypass of regulating protein functions. For example, both methylation and phosphorylation are able to trigger acetylation of histones [20].

Here, while introducing the physiological functions of $\mathrm{N}$ myristoylation, we also delineate the cross talk of $\mathrm{N}$ myristoylation components with signaling constituents in light of well-established studies to explore the robust role of $\mathrm{N}$ myristoylation in cell biology.

Dynamic structural changes in membrane anchoring and intracellular trafficking

One of the major functions of $\mathrm{N}$-myristoylation is to facilitate protein binding in membranes. In fact, Peitzsch and McLaughlin 
established a tenet stating that the myristoyl motif is insufficient for the stable anchorage of a protein to a lipid bilayer [21]. Therefore, a second signal, comprising a group of hydrophobic residues, positively charged amino acids or another lipid moiety, is required for stable membrane attachment.

In one scenario called the 'ligand-dependent switch' (Fig. 2a), the conformation of a protein is changed upon ligand binding, exposing the myristoyl motif that attaches to a component in the lipid bilayer. For example, the GTP-myristoyl switch facilitates the membrane interaction of ARF [22, 23]. The exposed myristoyl motif and the basic hydrophobic residues in the $\mathrm{N}$-terminus facilitate the interaction of ARF1-GTP with the membrane. The second scenario refers to a cluster of positively charged amino acids that are associated with a cofactor, such as calcium (Fig. 2b), or are phosphorylated (Fig. 2c); the former cluster accumulates a positive charge to strengthen membrane binding, while the latter attenuates the positive charge to weaken membrane binding and cause membrane dissociation. The binding of two calcium ions to EF-hand motifs in the recoverin protein facilitates the exposure of a myristoyl group from a hydrophobic cavity to solvent (Fig. 2b) [24]. Another example is the myristoylated alanine-rich $C$ kinase substrate (MARCKS) protein. The phosphorylation of serine residues contributes to its membrane dissociation, since the phosphate moiety reduces the positive charge (Fig. 2c) [25]. Ece C. Gaffarogullari et al. [26] proposed a novel myristoyl/phosphorylation switch in a PKA-C model of membrane attachment. PKA-C maintains conformational equilibrium between a myr-in state and a myr-out state, which refer to the myristoyl group tucked into the hydrophobic pocket of the PKA-C or extruded from the hydrophobic pocket, respectively. In the myr-out sate, the exposed myristoyl group inserts into the lipid bilayer and facilitates PKA-C binding to the membrane. Therefore, it was proposed that a large population of PKA-C maintains the myr-in state distal to the membrane and that PKA-C shifts to the myr-out state in the proximity of the plasma membrane or when phosphorylated at Ser10 independent of the regulatory subunit.

The palmitoyl moiety, which consists of a 16-carbon fatty chain, also induces signal transduction (Fig. 2d). It was observed that $\mathrm{H}$-Ras requires both a myristoyl group and palmitoyl group to target the membrane and stimulate kinase activity, whereas only $\mathrm{N}$-myristoylated $\mathrm{H}$-Ras acts a substrate for palmitoyltransferases and therefore is palmitoylated $[27,28]$. The $\mathrm{N}$-myristoylationnegative mutants of CDPK2 [29] and fibroblast growth factor receptor substrate $2 a$ (FRS2a) [30] do not incorporate palmitate and therefore do not attach to the plasma membrane.

Moreover, proteins with myristoyl moieties show a tendency for inclusion in the membrane fraction. For example, it was reported that both $\mathrm{N}$-myristoylation and palmitoylation appear to have opposing roles and different membrane lipid microdomain preferences for the $G$ protein-membrane interactions I (Gai1) monomer, which are likely due to the conformational differences in the presence of different fatty acids [31]. The Gai1 protein that is $\mathrm{N}$-myristoylated but not palmitoylated preferentially anchors to lamellar-prone membrane domains with a net negative charge. In contrast, the Gai1 protein that is both N-myristoylated and palmitoylated preferentially localizes to raft-like lamellar membranes without negative charges. N-myristoylated Akt1 (MyrAkt1) exhibits a distinct substrate preference that is not exhibited by the cytosolic or the membrane fractions that do not include lipid rafts, indicating that Akt1 for which oncogenicity is conferred by $\mathrm{N}$ myristoylation is enriched in lipid rafts [7]. In addition, the evidence that simvastatin or cholesterol depletion preferentially ablates the phosphorylation of MyrAkt1 in lipid rafts suggests that MyrAkt1 is modified in a cholesterol-sensitive manner (Fig. 2e).

Differential orientation of intracellular trafficking and localization It is well known that the most organelles in cells, such as the endoplasmic reticulum (ER), mitochondrion and endosome, are

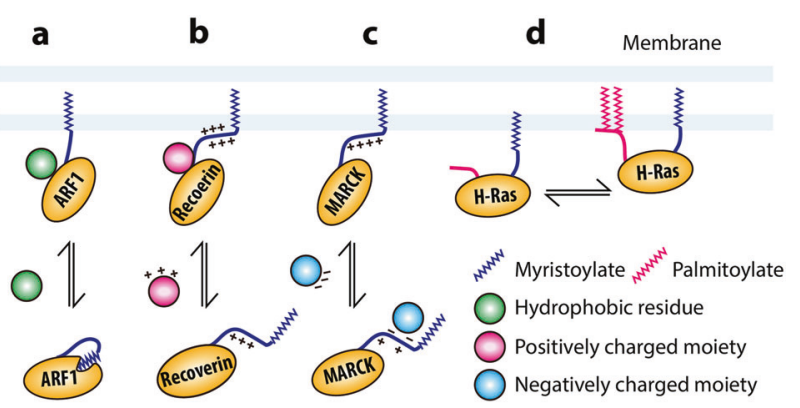

e
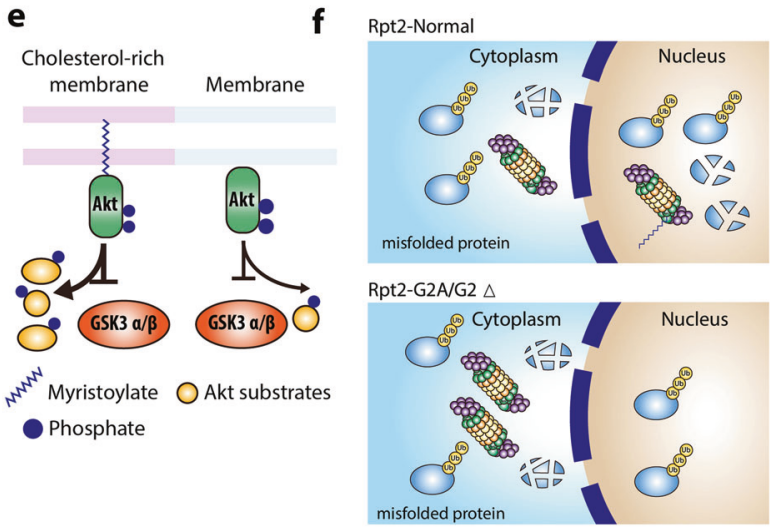

Fig. 2 Schematic showing N-myristoylation affecting protein binding to membranes and subcellular trafficking. a-d Schematic of the two-signal hypothesis of myristate-mediated membrane binding. e Phosphorylated Akt tends to accumulate in cholesterol-enriched cell membrane regions and actively stimulates downstream signaling. $\mathbf{f}$ In wild-type cells (upper panel), $\mathrm{N}$-myristoylation (dark blue) contributes to proteasomes shuttling between the cytoplasm and nucleus, which leads to misfolded proteins in the cytoplasm and nucleus being degraded by proteasomes. In Rpt2-G2A and Rpt-G2 $\Delta$ cells (lower panel), $\mathrm{N}$-myristoylation-deficient proteasomes are insufficiently transported to the nucleus resulting in the elevated accumulation of misfolded proteins in the nucleus

bound to the plasma membrane and that these membrane interactions produce different effects. In addition, some proteins must undergo $\mathrm{N}$-myristoylation for subcellular trafficking and localization. Some mitochondria-related proteins, such as TOMM40, SAMM50 [32] and, CLPABP [33], were demonstrated to require the function of myristoylated proteins to bind to the mitochondrial outer membrane. In one mechanism, the hydrophobic myristoyl group motif increases dependence of the protein to reduce cytoplasmic shuttling. For example, in mice, binding domain-containing protein 1 (Stbd1) was observed to be a transmembrane resident protein, and nonmyristoylated Stbd1 was shuttled with ease between the ER and mitochondria [34]. Ring finger protein 11 (RNF11) colocalizes with both early and recycling endosomes. While RNF11 requires N-myristoylation and Spalmitoylation for membrane binding, $\mathrm{N}$-myristoylation plays a greater role [35]. The removal of myristoylate results in protein diffusion. In another mechanism, the hydrophobicity of the myristoyl moiety increases protein flexibility, facilitating its shuttling through hydrophobic areas. In a yeast model [36], the proteasome subunit Rpt2 is cotranslationally N-myristoylated. Loss of Rpt2 N-myristoylation causes abnormal dynamic nucleuscytoplasm translocation of proteasomes and the aberrant accumulation of ubiquitinated protein levels (Fig. $2 \mathrm{f}$ ).

Requirements for protein assembly and protein-protein interaction

Many proteins require assembly for maturity and function, and some evidence indicates that $\mathrm{N}$-myristoylation drives the 
aggregation of proteins in some cases [37]. Spassov et al. reported that Src dimerization is mediated by the myristoylated $\mathrm{N}$-terminal region of one partner interacting with the hydrophobic kinase domain of its counterpart [38, 39]. Both Y419 autophosphorylation and dimerization are codependent and activate Src kinases (Fig. 3a). The functions of Src kinases are disrupted by interfering dimerization, emphasizing the importance of $\mathrm{N}$-myristoylation to Src activity. In addition, the myristoyl-group-driven aggregation in lipid bilayers is also observed for H-Ras [37].

Some protein-protein interactions require a myristoyl group. The brain-specific protein CAP-23/NAP22 binds calmodulin (CaM) with high affinity even though it lacks any canonical CaM-binding domain. A crystal structure of $\mathrm{Ca}^{2+}$-CaM-CAP-23/NAP22-CaM complex showed that the myristoyl group of CAP-23/NAP22 passes through a hydrophobic tunnel created by two hydrophobic components exclusively in the pockets of the terminus of CaM, implying the direct involvement of the myristoyl group in this interaction. Further, the interaction of calmodulin induces the phosphorylation of CAP-23/NAP-22 [40].

In HIV infections, both Nef and Gag are myristoylated by the host NMT cell to execute proper functions. In the assembly of HIV, Nef and Gag proteins are essential for infection. Nef has many virulence factors that attenuate the immune system recognition of infected cells and enhance infectivity and viral replication [12], and Gag is a precursor protein for structural components of the viral capsid. Because this virus lacks NMT proteins, both Nef and Gag are myristoylated by the host NMT cell, and the Gag-Gag interaction triggers an entropic switch toward a myristoyl-exposed conformation, providing the impetus for protein assembly (Fig. 3b). In contrast, in beta-retroviruses, such as mouse mammary tumor virus (MMTV), the oligomerization of the matrix (MA) domain, which contains the N-terminal residue in Gag, adopts a myristoylsequestering conformation [41, 42]. Moreover, the results from screens of the interaction of the host factors with the HIV-1 MA domain showed that heme oxygenase $2(\mathrm{HO}-2)$ specifically binds the myristoyl moiety of Gag via a hydrophobic channel adjacent to its heme-binding pocket, which inhibits virus production. In addition, HO-2 binds N-myristoylated TRAM, an adaptor protein for Toll-like receptor 4 (TLR4) [43], which inhibits the TRAMdependent lipopolysaccharide(LPS)-TLR4 pathway. These findings suggest $\mathrm{HO}-2$ is a novel cellular $\mathrm{N}$-myristoylated protein that negatively regulates both virus replication and host inflammatory responses [44].

Regulation of protein stability

Recent studies have revealed that a glycine positioned in the $\mathrm{N}$ terminus can act as a potent degron, indicating that $\mathrm{N}$ myristoylation may contribute to the removal of proteolytic cleavage products. Richard T. Timms et al. [45] identified two Cul2 cullin-RING E3 ubiquitin ligase complexes called Cul2 $2^{\text {ZYG11B }}$ and Cul2 ${ }^{\text {ZER1 }}$, both of which target $\mathrm{N}$-myristoylated proteins for proteasomal degradation by recognizing $\mathrm{N}$-terminal glycine degrons, which presumably play important roles in the quality control of protein $\mathrm{N}$-myristoylation. Nonmyristoylated c-Src has enhanced stability compared to that of soluble $\mathrm{N}$-myristoylated cSrc [46].

Borja Belda-Palazon et al. [47] found that RGLG1, an E3 ligase in Arabidopsis, is N-myristoylated by NMT1. N-myristoylation facilitates the attachment of RGLG1 to the plasma membrane. The phytohormone abscisic acid ( $A B A$ ) induces the degradation of PP2CA, which is predominantly localized in the nucleus, through RGLG1/5 E3 ligases. This degradation mechanism was found by ABA downregulation of NMT1, which hindered the $\mathrm{N}$ myristoylation of RGLG1 and promoted its translocation to the nucleus, where it interacted with PP2CA, increasing PP2CA degradation. In addition, in Aspergillus nidulans [48], the swoF gene was found to encode an NMT. The enhanced activity of the $26 \mathrm{~S}$ proteasome and the accumulation of ubiquitinated substrates a

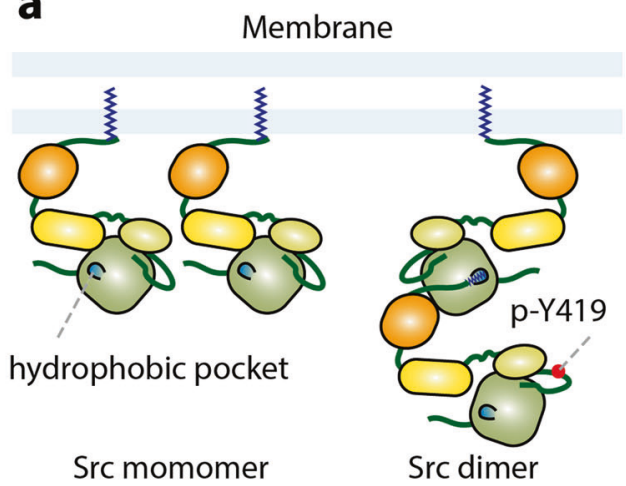

b Membrane
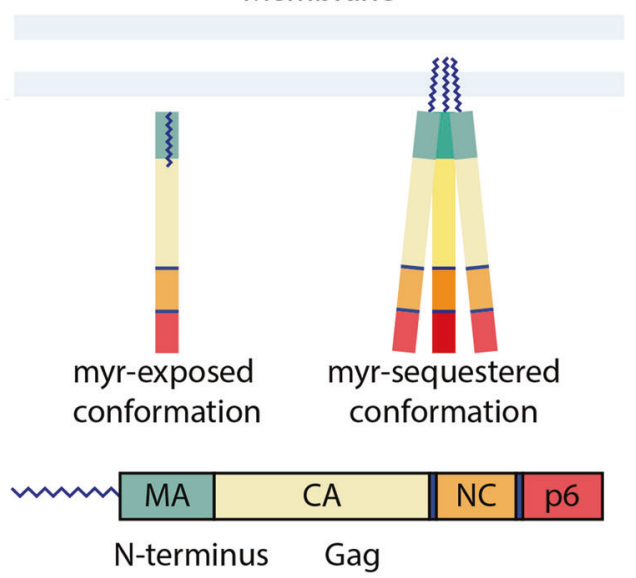

Fig. 3 Schematic representation of the impacts of N-myristoylation on protein assembly. myr refers to myristoyl moiety

in the $\mathrm{N}$-myristoylate-deficient swoF mutant, compared with the activity and accumulation in wild-type strain, resulted in impaired cell morphogenesis.

Alterations to signaling pathways

$\mathrm{N}$-myristoylation influences downstream kinase activity directly or indirectly, usually by the mechanisms described above, through Ras and Src. c-Abl is a member of the Src family of tyrosine kinases. A 'myristoyl/phosphotyrosine' switch has been identified in the regulation of the kinase activity of c-Abl [49]. Nmyristoylation locks the protein into an autoinhibitory conformation when the $\mathrm{SH} 2$ domain docks to the kinase domain. In contrast, myristoylation leads to an unpredicted function: c-Src is induced into a conformation optimal for kinase activity. A series of studies have unequivocally demonstrated that $\mathrm{N}$-myristoylation of the $\beta$-subunit is a prerequisite for the initiation of AMPK signaling in response to AMP $[26,50,51]$. Therefore, in the case of NMT deficiency or upon cell treatment with an NMT inhibitor, suppressed $\mathrm{N}$-myristoylation diminished the extent of $\mathrm{a}$-Thr172 phosphorylation of AMP and abolished its activation, thereby causing multiple morbid physiological outcomes. Moreover, the myristoyl switch regulated by AMP may affect the selectivity of the substrates and serve as a gatekeeper for transducing signals of metabolic stress [52].

Recently, it was suggested that PKA-C N-myristoylation may account for the enriched kinase activity at the membrane and the preferential phosphorylation of membrane substrates. These findings indicate an important role for $\mathrm{N}$-myristoylation in synaptic function normality and plasticity during normal PKA regulation $[53,54]$. 
In another pattern of results from recent studies, several proteins were found to phosphorylate NMT, resulting in the activation of NMT. Rajala et al. [55] verified that Lyn and Fyn are Nmyristoylated by NMT before they phosphorylate NMT. In addition, it has been hypothesized that the interaction between NMT1 and the Lyn tyrosine kinase proceeds in a phosphorylationdependent manner [56].

\section{Manipulation of substrates during apoptosis}

Apoptosis, or programmed cell death, is the physiologically and tightly controlled process for eliminating unnecessary or redundant cells in eukaryotic organisms. In addition, dysfunctional apoptosis is one of the characteristics of malignant cells. Apoptosis is orchestrated by a signaling cascade of proteases called caspases (cysteine-containing aspases), which leads to the exposure of novel $\mathrm{N}$-termini susceptible to various posttranslational modifications. Since the initial discovery of myristoylated Bid, it has been revealed that multiple proteins are modified posttranslationally by $\mathrm{N}$-myristoylation upon caspase cleavage. Using a myristoylated protein-labeling method in Jurkat $\mathrm{T}$ cells and MCF-7 cells, at least 15 and 7 posttranslationally $\mathrm{N}$ myristoylated proteins were discovered, respectively, during apoptosis [57]. Further characterization of these proteins revealed that caspase truncated (ct)-gelsolin and ct-PKC have antiapoptotic roles while ct-Bid and ct-PAK2 have proapoptotic roles upon posttranslational $\mathrm{N}$-myristoylation, suggesting that posttranslational $\mathrm{N}$-myristoylation plays an important role in maintaining a balance between cell death and survival. Moreover, it has been found that NMT1 and NMT2 are substrates of different caspases.

Furthermore, N-myristoylation may be involved in sphingolipid biosynthesis pathway, which includes ceramide synthesis and is involved in the induction of apoptosis. In the last step of ceramide biosynthesis, a trans $\triangle 4$-double bond in the carbon chain of dihydroceramide is specifically catalyzed by dihydroceramide $\triangle 4$ desaturase 1 (DES1), which is N-myristoylated by NMT1 [58]. Myristic acid upregulates novel DES1 activity, which differs from that of saturated fatty acids, possibly because of the accumulation of N-myristoylated DES1. N-myristoylation causes a proportion of DES1 to be translocated from the ER to the mitochondrial outer membrane, leading to an increase in ceramide levels. Since the production of ceramide can induce apoptosis by inducing mitochondrial dysfunction [59], the myristic acid-associated increase in DES1 activity can enhance apoptosis induction in cells, which shows the importance of $\mathrm{N}$-myristoylation in the process of apoptosis. N-myristoylated LANCL2, which belongs to the eukaryotic LanC-like protein family, is known as the testisspecific adriamycin sensitivity protein (TASP). One biochemical study identified a phosphatidylinositol phosphate (PIP)-binding site in the N-terminus of LANCL2. In addition, both $\mathrm{N}$ myristoylation and PIP binding are crucial for LANC2 binding to the membrane. It is possible that the overexpression of LANCL2 increases cell sensitivity to adriamycin, which is dependent on both $\mathrm{N}$-myristoylation and membrane association [60].

In summary, we can conclude that a myristoyl group as a hydrophobic motif always stably binds to substrates to change the conformation and increase the hydrophobicity of the protein. Further, it affects protein localization and the ease with which a protein binds to substrates. However, insufficient hydrophobicity of the 14-carbon myristoyl group leads to it being influenced by its surroundings. The hydrophilic group can ease protein binding, and the hydrophobic group can stabilize the state of the N-myristoylated protein. It seems that $\mathrm{N}$-myristoylation triggers consequential functions of protein necessarily but insufficiently.

\section{MULTIPLE USES OF NMT INHIBITORS IN TREATING DISEASE}

Accumulating evidence has demonstrated that $\mathrm{N}$-myristoylation is an evolutionarily conserved lipidation that is essential for cell viability in different organisms. Currently, interventions of protein $\mathrm{N}$-myristoylation are principally achieved through NMT inhibitors. Therefore, NMT inhibitors are very suitable for use against diseases caused by proliferating cells or pathogens, such as infectious diseases caused by various pathogens and malignancies. In fact, broadly classified inhibitors for each process in NMT-induced disease are designated as antifungal and antiviral agents. Typically, these inhibitors can be divided into three categories [56]: (1) Myristoyl-CoA and myristate derivatives. Although the myristoyl-CoA binding sites in human NMT and fungal NMT are highly conserved, their peptide-binding sites are divergent, which provides an explanation for the selectivity of inhibitors that do not induce adverse toxicity in humans. (2) Histidine analogs. In most conserved regions in the catalytic domain EEVEH (289-293), histidine is critical for the myristoyl-CoA transfer [61]. (3) Myristoyl peptide derivatives. As previously mentioned, lipid metabolism disorders can affect protein lipidation [1]. Various saturated and unsaturated fatty acids have been evaluated as potent inhibitors of human NMT1.

Indeed, the development of NMT inhibitors is not limited to proteins involved in infectious diseases because they are great prospects as immunodeficiency and cancer treatments.

\section{Hematopoiesis and immunodeficiency}

As mentioned previously, NMT1 has been characterized as the principal enzyme for the early development of embryogenesis. Further investigation was focused on the role of NMT1 in myelopoiesis through which blood cell types are matured. Bone marrow-derived macrophages (BMDMs) from $\mathrm{NMT}^{+/-}{ }^{+-}$-deficient mice have defective morphology compared with that of mature BMDMs in wild-type mice. During BMDM maturation, the NMT activity increased during the initial period and then decreased for the remaining time due to differential NMT expression. Although the NMT activity in the BMDMs of $\mathrm{NMT}^{+/-}$-deficient mice followed a trend similar to that of the BMDMs in the wild-type mice, the NMT expression levels were reduced to approximately one-half in the mutant mice. This report addressed an essential role of NMT1 during monocytic/macrophage differentiation.

Of course, studies are continuously uncovering valuable evidence not only for NMT1 but also for NMT2 in both normal and malignant hematopoiesis. A bioinformatic database of gene expression in different cancer cell lines revealed decreased NMT2 expression and preserved NMT1 expression in some hematological cancer cell lines, such as Burkitt lymphoma, diffuse large B-cell lymphoma and acute myeloid leukemia (AML) cell lines. A study performed by Ryan Stubbins et al. suggested a correlation between NMT2 and AML. NMT1 and NMT2 protein levels in the marrow aspirates or peripheral blood of $A M L$ patients were assayed by fluorescence-activated cell counting with intracytoplasmic staining, expressed as the mean fluorescent intensity (MFI). The evidence showed that the NMT2 MFI was higher in the lymphocytes and lower in the monocytes, suggesting that the regulation of NMT2 protein levels may influence early lymphoid/myeloid lineage commitment. Further, the overall trend revealed by a survival analysis showed higher NMT2 MFI values, portending a worse prognosis for AML patients and suggesting a role for NMT2 as a novel prognostic biomarker for intermediate-risk AML [62].

In terms of adaptive immunity, it has been reported that $\mathrm{N}$ myristoylation is indispensable for T cell development $[63,64]$. A comparative analysis of thymuses showed that mice with deficient NMT1 or NMT2 levels had reduced medullary volume, which was $30 \%$ and $25 \%$ lower, respectively, than it was in wild-type mice, and mice with double NMT1 and NMT2 deficiency had a much greater medullary volume reduction (83\%). These findings suggest nonredundant roles for both NMTs in maintaining the development of thymocytes, since the thymus has high NMT activity levels [65]. In agreement with these results, it was demonstrated that 
T-cell receptor (TCR) signaling is disrupted in mice with T-cell lineages characterized by specific NMT1 and NMT2 activity deficiencies and results in retarded thymocyte development. These outcomes may be attributable to the mislocalization of $\mathrm{N}$ myristoylation-deficient Src family tyrosine kinases, such as Lck or Fyn [66]. For example, $\mathrm{N}$-myristoylation contributes to the cytomembrane targeting of Fyn and facilitates its binding with the $z$ chain of TCR. Non-N-myristoylated LCk is in the cytoplasm and unable to facilitate TCR signaling. However, the regulation of $\mathrm{N}$-myristoylation or NMT activity in these processes during adaptive immunity has not been well established to date. To gain in-depth understanding of the regulation of immune responses, further investigation into the specific roles of $\mathrm{N}$ myristoylation, which may involve NMT as a potential target of immune modulation, is warranted.

Parasitic and other infectious diseases

It is known that some $\mathrm{N}$-myristoylated proteins in small RNA viruses and retroviruses are essential for virus assembly during viral replication or production of infectious viral particles, suggesting that $\mathrm{N}$-myristoylation may be related to the survival and propagation of pathogens. In addition, some pathogens need to utilize host cellular machinery to replicate within host cells due to deficiency in viral NMTs. In immunosuppressed patients, Cryptococcus neoformans can easily cause chronic meningitis; however, it is unable to survive at $37^{\circ} \mathrm{C}$ if it harbors mutant NMT with reduced activity. Some disease-causing parasitic protozoa such as falciparum (malaria), Leishmania donovani (leishmaniasis), and Trypanosoma brucei (African sleeping sickness) retain the NMT necessary for their survival. Given that the peptide-binding pocket of NMT is not strictly conserved across species, the search for species-specific NMT inhibitors focused on the binding pocket is worthwhile [67].

Notwithstanding, current anti-infectious agents have some drawbacks, such as drug resistance, poor oral bioavailability, and renal toxicity. A group of studies have suggested NMT as a novel target for use in anti-infective drugs. Theoretically, according to the respective skeletal structures, NMT inhibitors are classified into four categories (Table 1). Among these inhibitors, benzofuran and, benzothiazole have high species selectivity [68].

Anti-infective drugs targeting NMT have certain advantages. First, myristoyl-CoA is found at very low accumulation levels, at approximately $5 \mathrm{nM}$, in mammalian cells. In addition, the strict hydrophobic structure of the fatty carbon chains reduces the likelihood that incompatible fatty acid groups will be recognized by the NMT, even in cases where the palmitic acid concentration is higher than the myristic acid in vitro [69]. These physiological phenomena suggest that a small amount of inhibitor can have a good inhibitory effect. Second, NMT inhibitors can be designed for high selectivity because of the significant differences in substrate specificity in the human and parasitic organisms. Moreover, many fungal and parasites must use the NMT of the host to synthesize essential proteins for their own reproduction.

\section{Potential targets of cancer treatments}

Given that altered NMT expression is observed in many types of cancer tissues and because many $\mathrm{N}$-myristoylated proteins are involved in signaling processes that regulate cell proliferation, growth and death, it has been proposed that $\mathrm{N}$-myristoylation or NMTs can be considered as therapeutic targets for cancer. The premise for their use is based on a thorough understanding of the abnormal regulation of $\mathrm{N}$-myristoylation in carcinogenesis. For instance, given that $\mathrm{N}$-myristoylation can facilitate Src-mediated prostate tumorigenesis, the myristoyl-CoA analog B13 and its derivative LCL204 $[68,70]$ have been identified as inhibitors of NMT1 enzymatic activity and blockers of Src N-myristoylation; their actions are based on competing for myristoyl-CoA binding site, which provides a promising approach to inhibit Src family kinase-mediated oncogenic activity, and offers preclinical support for the use of protein $\mathrm{N}$-myristoylation inhibitors in treating cancer [71]. An organopalladium compound, Tris(dibenzylideneacetone) dipalladium (Tris DBA) was identified as a novel human NMT1 inhibitor that not only blocks the kinase activity of NMT1 but also reduces its expression. It showed potent antiproliferative activity against melanoma cells by inhibiting several proliferation-related signaling pathway proteins including MAPK, Akt, and STAT-3 [72].

The most successful examples of myristoylation-related anticancer inhibitors are allosteric Abl TKIs such as ABL001 [73-75], GNF2 and GNF5 [76] (Table 1). These allosteric inhibitors selectively target the myristoyl-binding pocket in the C-lobe of the Abl kinase domain and not the ATP-binding pocket. Moreover, these inhibitors increase the sensitivity of a Bcr-Abl (T315I) mutant to ATP-competitive TKIs. A series of phase I clinical trials of ABL001 and TKIs (nilotinib, imatinib, and dasatinib) therapy have been ongoing for $\mathrm{CML}$ and $\mathrm{Ph}+\mathrm{ALL}$ patients (https://clinicaltrials.gov/ ct2/show/NCT02081378).

Some studies have shown the potential targets of NMT in cancer cells. The Cancer Genome Atlas (TCGA) reports a group of genomic alterations induced by NMT1 and NMT2 in several cancers. Although the occurrence of somatic mutations is more common than the occurrence of genomic amplification in NMT1 and NMT2, the roles of these mutations in regulating pathological mechanisms remain to be determined (https://www.cbioportal. org/). Moreover, patients with diseases such as liver cancer, cervical cancer, and lung cancer and high expression levels of NMT1 or NMT2 are more likely to have a poor prognosis, as indicated consistently in some reports. In addition, it is noteworthy that a cohort with renal cell carcinoma with either high expression of NMT1 or low expression of NMT2 was found to have worse overall survival, suggesting the unique roles of these proteins in kidney cancer. However, no evidence showed that their catalytic activity is linked to tumor progression in kidney cancer (http:// kmplot.com/analysis/).

\section{NMT kinase activators}

In addition, several NMT activators that enhance NMT enzyme activity have been identified. L-histidine and $D$-histidine can activate human NMT activity in a concentration-dependent manner. This finding suggests that two analogs may be involved in myristoyl-CoA transfer by interacting with His-293 of NMT. NAF45 was identified as an NMT activator factor in bovine brain [77]. The reconstitution of NAF45 and NMT likely resulted in an open conformation where the active site is expanded. A novel stratagem of NMT activation is enhanced NMT specificity for specific myristoyl-CoA substrates. On the basis that NMT can transfer abundant palmitoyl-CoA but can use only the rare myristoyl-CoA for acylation of a substrate protein, Eric Soupene et al. [78] determined that the acyl-CoA-binding protein domain 6 (ACBD6) stimulates the activity of NMT2. ACBD6 can block the access of palmitoyl-CoA to the NMT2-binding site and enhance its catalytic activity, which requires interaction between NMT2 and ACBD6. A mutant ACBD6 unable to interact with NMT2 or with deficient ligand binding at its own $\mathrm{N}$-terminus does not stimulate NMT2 activity.

\section{CONCLUSION AND PERSPECTIVE}

In this review article, we outlined advanced studies of $\mathrm{N}$ myristoylation, focusing on the role of protein $\mathrm{N}$-myristoylation in physiology and pathology. The important role of $\mathrm{N}$ myristoylation underlies the early stages of protein maturation. After the protein is folded in the Golgi apparatus or the ER, cotranslationally $\mathrm{N}$-myristoylation is likely to influence the transport and localization of the protein, which can affect the biofunction of protein. The insufficient NMT kinase activity in pathogens and the variations in different species emphasize the 
Table 1. Chemical structure and in vitro pharmacological properties of different types of NMT inhibitors

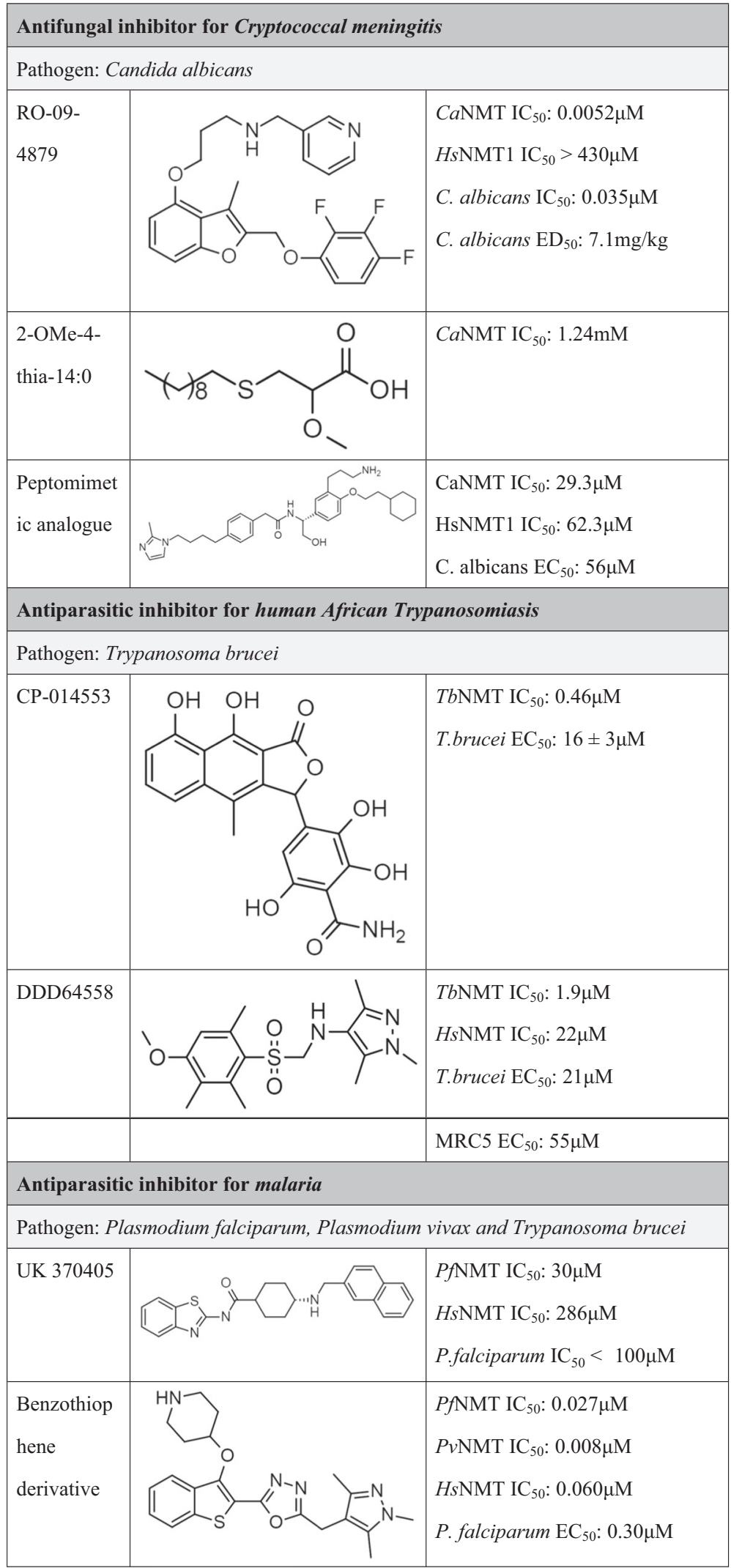




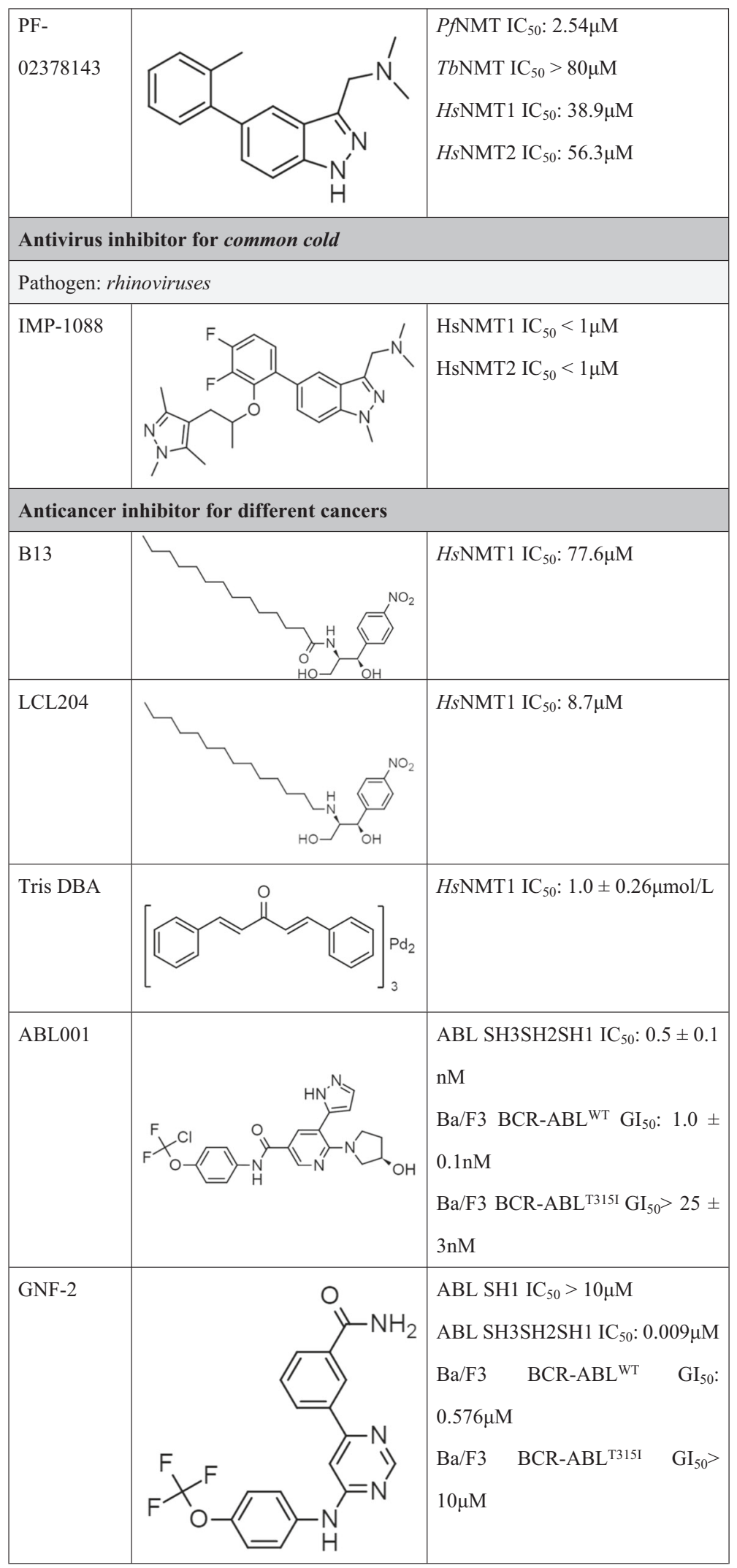




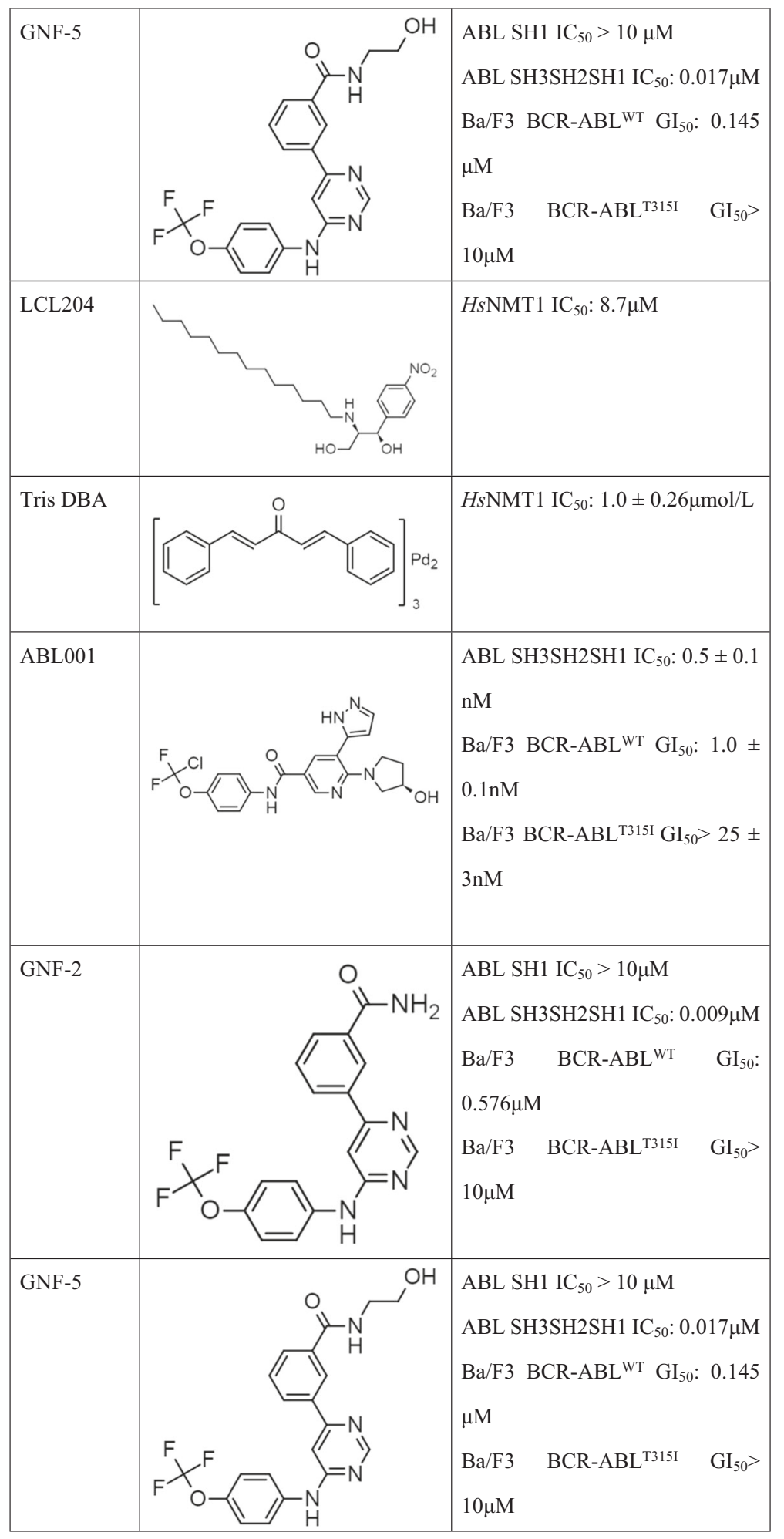

$\mathrm{ABL}$ SH1 refers to the short $\mathrm{ABL}$ construct only contains ATP kinase domain. ABL SH1SH2SH3 refers to the long ABL construct contains ATP kinase domain and myristate binding domain

CaNMT Candida albicans NMT, HsNMT1 human NMT1, TbNMT Trypanosoma brucei NMT, PfNMT $P$. falciparum NMT, IC $C_{50}$ half inhibitory concentration, $\mathrm{EC}_{50}$ half maximal effective concentration, $\mathrm{Gl}_{50}$ values from cellular proliferation assay in $\mathrm{Ba} / \mathrm{F} 3 \mathrm{BCR}-\mathrm{ABL} 1$ dependent cells 
selective lethality of NMT inhibitors for infectious diseases for which either no valid drug is qualified or for which available drugs induce drug resistance. The breakthroughs of NMT inhibitors will likely be as tumor treatments. ABL001 is being pioneered continuously for CML and $\mathrm{Ph}+\mathrm{ALL}$ patients in phase I trials, which suggests a strategy focused on the N-myristoylation of oncoproteins. Furthermore, posttranslational N-myristoylation in the apoptotic process suggests the participation of NMTs, specifically NMT2, in cell death. The function of the N-myristoylated protein in the apoptotic process, whether pathogenically or physiologically normal, can further indicate the orientation of the treatment strategy for targeting NMT.

The knowledge of other protein modifications, such as ubiquitination or palmitoylation, which involves a multimember kinase family, can inform many specific enzyme-substrate studies and the design of selective inhibitors. In contrast, scientists need more precise approaches for analyses of NMTs and Nmyristoylation. Nevertheless, traditional methods based on radioactively labeled probes to detect $\mathrm{N}$-myristoylation are insensitive and time consuming, and the technical difficulties in obtaining three-dimensional structures of myristate-attached proteins need to be overcome. Further, bioinformatics on $\mathrm{N}$-myristoylation and NMTs is still in its infancy; however, since databases are integrated, these data provide many clues linked to other biological fields.

Regardless of the technical difficulties, it is worth exploiting novel NMT inhibitors as single agents and exploring the potential drug synergies that might improve multiple clinical applications and enhance therapeutic efficacy, reverse drug resistance, or extend the therapeutic index for drugs already used in the clinic. It is likely that two approaches [79] can be used to reveal the prospects of NMT inhibitors for oncology therapy in the future: (1) identify currently unknown sensitivities of certain cancer types by widely screening cancer cell line panels and (2) discover the essential sensitivity or resistance mechanisms in resistant cell lines and wild-type cell lines by proteomic analyses of NMT substrate profiles and proteomic changes. As our knowledge of the biochemistry and cell biology of $\mathrm{N}$-myristoylation continues to grow, more substrate proteins will be identified, and scientists will continue to deduce the effects of this lipid attachment on protein structure and function.

\section{ACKNOWLEDGEMENTS}

This work was supported by grants from the National Natural Science Foundation of China (81872885 to J.C.), Zhejiang Provincial Natural Science Foundation (Y18H310005 to J.C.), and the Talent Project of Zhejiang Association for Science and Technology (No. 2018YCGC002 to J.C.).

\section{AUTHOR CONTRIBUTIONS}

MY and JC conceived and designed the review article. MY, ZHS, and JC collected the related research articles contributed to the paper. MY, MDY, HZ, BY, QJH, and JC made amendments to the paper.

\section{ADDITIONAL INFORMATION}

Competing interests: The authors declare that the research was conducted in the absence of any commercial or financial relationships that could be construed as potential competing interests.

\section{REFERENCES}

1. Chen BE, Sun Y, Niu JX, Jarugumilli GK, Wu X. Protein lipidation in cell signaling and diseases: function, regulation, and therapeutic opportunities. Cell Chem Biol. 2018;25:817-31.

2. Nadolski MJ, Linder ME. Protein lipidation. FEBS J. 2007;274:5202-10.

3. Jing $H$, Zhang $X$, Wisner SA, Chen $X$, Spiegelman NA, Linder ME, et al. SIRT2 and lysine fatty acylation regulate the transforming activity of K-Ras4a. Elife. 2017;6:1-32.
4. Cao J, Sun L, Aramsangtienchai $P$, Spiegelman NA, Zhang $X$, Huang $W$, et al. HDAC11 regulates type I interferon signaling through defatty-acylation of SHMT2. Proc Natl Acad Sci U S A. 2019;116:5487-92.

5. Lin HN, Su XY, He B. Protein lysine acylation and cysteine succination by intermediates of energy metabolism. Acs Chem Biol. 2012;7:947-60.

6. Wright MH, Heal WP, Mann DJ, Tate EW. Protein myristoylation in health and disease. J Chem Biol. 2010;3:19-35.

7. Adam RM, Mukhopadhyay NK, Kim J, Di Vizio D, Cinar B, Boucher K, et al. Cholesterol sensitivity of endogenous and myristoylated Akt. Cancer Res. 2007;67:6238-46.

8. Hu T, Li C, Cao Z, Van Raay TJ, Smith JG, Willert K, et al. Myristoylated Naked2 antagonizes Wnt-beta-catenin activity by degrading Dishevelled-1 at the plasma membrane. J Biol Chem. 2010;285:13561-8.

9. Schwertassek U, Buckley DA, Xu CF, Lindsay AJ, McCaffrey MW, Neubert TA, et al. Myristoylation of the dual-specificity phosphatase c-JUN N-terminal kinase (JNK) stimulatory phosphatase 1 is necessary for its activation of JNK signaling and apoptosis. FEBS J. 2010;277:2463-73.

10. Tan YW, Hong WJ, Chu JJ. Inhibition of enterovirus VP4 myristoylation is a potential antiviral strategy for hand, foot and mouth disease. Antivir Res. 2016;133:191-5.

11. Kumar S, Sharma RK. N-Terminal region of the catalytic domain of human Nmyristoyltransferase 1 acts as an inhibitory module. PLos ONE. 2015;10:1-20.

12. Takamune N, Gota K, Misumi S, Tanaka K, Okinaka S, Shoji S. HIV-1 production is specifically associated with human NMT1 long form in human NMT isozymes. Microbes Infect. 2008;10:143-50.

13. Glover CJ, Hartman KD, Felsted RL. Human N-myristoyltransferase amino-terminal domain involved in targeting the enzyme to the ribosomal subcellular fraction. J Biol Chem. 1997;272:28680-9.

14. Tang HY, Han M. Fatty acids regulate germline Sex determination through ACS-4dependent myristoylation. Cell. 2017;169:457-69.

15. Lodge JK, Johnson RL, Weinberg RA, Gordon Jl. Comparison of myristoyl-CoA: protein N-myristoyltransferases from three pathogenic fungi: Cryptococcus neoformans, Histoplasma capsulatum, and Candida albicans. J Biol Chem. 1994;269:2996-3009.

16. Ducker CE, Upson JJ, French KJ, Smith CD. Two N-myristoyltransferase isozymes play unique roles in protein myristoylation, proliferation, and apoptosis. Mol Cancer Res. 2005;3:463-76.

17. Teng $Y B$, Jing $H$, Aramsangtienchai $P, H e B$, Khan $S$, Hu J, et al. Efficient demyristoylase activity of SIRT2 revealed by kinetic and structural studies. Sci Rep.-Uk. 2015;5:1-8.

18. Zhang XY, Spiegelman NA, Nelson OD, Jing $H$, Lin HN. SIRT6 regulates Ras-related protein R-Ras2 by lysine defatty-acylation. Elife. 2017;6:1-16.

19. Yang SH, Shrivastav A, Kisinski C, Sharma RK, Chen MH, Berthiaume LG, et al. Nmyristoyltransferase 1 is essential in early mouse development. J Biol Chem. 2005;280:18990-5.

20. Darieva Z, Webber A, Warwood S, Sharrocks AD. Protein kinase C coordinates histone $\mathrm{H} 3$ phosphorylation and acetylation. Elife. 2015;4:1-15.

21. Seykora JT, Myat MM, Allen LAH, Ravetch JV, Aderem A. Molecular determinants of the myristoyl-electrostatic switch of MARCKS. J Biol Chem. 1996;271: 18797-802.

22. Goldberg J. Structural basis for activation of ARF GTPase: mechanisms of guanine nucleotide exchange and GTP-myristoyl switching. Cell. 1998;95:237-48.

23. Liu YZ, Kahn RA, Prestegard JH. Structure and membrane interaction of myristoylated ARF1. Structure. 2009;17:79-87.

24. Tanaka T, Ames JB, Harvey TS, Stryer L, Ikura M. Sequestration of the membranetargeting myristoyl group of recoverin in the calcium-free state. Nature. 1995:376:444-7.

25. Braun T, Mcllhinney RAJ, Vergeres G. Myristoylation-dependent N-terminal cleavage of the myristoylated alanine-rich $C$ kinase substrate (MARCKS) by cellular extracts. Biochimie. 2000;82:705-15.

26. Gaffarogullari EC, Masterson LR, Metcalfe EE, Traaseth NJ, Balatri E, Musa MM, et al. A myristoyl/phosphoserine switch controls CAMP-dependent protein kinase association to membranes. J Mol Biol. 2011;411:823-36.

27. Utsumi T, Sakura N, Nakano K, Ishisaka R. C-terminal 15 kDa fragment of cytoskeletal actin is posttranslationally $\mathrm{N}$-myristoylated upon caspase-mediated cleavage and targeted to mitochondria. FEBS Lett. 2003;539:37-44.

28. Cadwallader KA, Paterson H, Macdonald SG, Hancock JF. N-Terminally myristoylated ras proteins require palmitoylation or a polybasic domain for plasmamembrane localization. Mol Cell Biol. 1994;14:4722-30.

29. Martin ML, Busconi L. Membrane localization of a rice calcium-dependent protein kinase (CDPK) is mediated by myristoylation and palmitoylation. Plant J. 2000;24:429-35.

30. Barylko B, Chen YJ, Hennen J, Angert I, Chen Y, Mueller JD, et al. Myristoylationdependent palmitoylation of the receptor tyrosine kinase adaptor FRS2alpha. Biochem-Us. 2019;58:2809-13. 
31. Alvarez R, Lopez DJ, Casas J, Llado V, Higuera M, Nagy T, et al. G proteinmembrane interactions I: Galphai1 myristoyl and palmitoyl modifications in protein-lipid interactions and its implications in membrane microdomain localization. Biochim Biophys Acta. 2015;1851:1511-20.

32. Utsumi T, Matsuzaki K, Kiwado A, Tanikawa A, Kikkawa Y, Hosokawa T, et al. Identification and characterization of protein $\mathrm{N}$-myristoylation occurring on four human mitochondrial proteins, SAMM50, TOMM40, MIC19, and MIC25. Plos One. 2018;13:1-27.

33. Maeda A, Uchida M, Nishikawa S, Nishino T, Konishi H. Role of N-myristoylation in stability and subcellular localization of the CLPABP protein. Biochem Biophys Res Commun. 2018;495:1249-56.

34. Demetriadou A, Morales-Sanfrutos J, Nearchou M, Baba O, Kyriacou K, Tate EW, et al. Mouse Stbd1 is N-myristoylated and affects ER-mitochondria association and mitochondrial morphology. J Cell Sci. 2017;130:903-15.

35. Santonico E, Belleudi F, Panni S, Torrisi MR, Cesareni G, Castagnoli L. Multiple modification and protein interaction signals drive the Ring finger protein 11 (RNF11) E3 ligase to the endosomal compartment. Oncogene. 2010;29:5604-18.

36. Kimura A, Kurata Y, Nakabayashi J, Kagawa H, Hirano H. N-Myristoylation of the Rpt2 subunit of the yeast $26 \mathrm{~S}$ proteasome is implicated in the subcellular compartment-specific protein quality control system. J Proteom. 2016;130:33-41.

37. Li HL, Gorfe AA. Aggregation of lipid-anchored full-length H-Ras in lipid bilayers: simulations with the MARTINI force field. Plos One. 2013;8:1-11.

38. Spassov DS, Ruiz-Saenz A, Piple A, Moasser MM. A dimerization function in the intrinsically disordered N-terminal region of Src. Cell Rep. 2018;25:449-63.

39. Le Roux AL, Mohammad IL, Mateos B, Arbesu M, Gairi M, Khan FA, et al. A myristoyl-binding site in the $\mathrm{SH} 3$ domain modulates c-Src membrane anchoring. Iscience. 2019;12:194-203.

40. Matsubara $M$, Nakatsu $T$, Kato $H$, Taniguchi $H$. Crystal structure of a myristoylated CAP-23/NAP-22 N-terminal domain complexed with Ca2+/calmodulin. Embo J. 2004;23:712-8.

41. Resh MD. A myristoyl switch regulates membrane binding of HIV-1 Gag. Proc Natl Acad Sci U S A. 2004;101:417-8.

42. Dolezal M, Zabransky A, Dostal J, Vanek O, Brynda J, Lepsik M, et al. Myristoylation drives dimerization of matrix protein from mouse mammary tumor virus. Retrovirology. 2016;13:1-15.

43. Rowe DC, McGettrick AF, Latz E, Monks BG, Gay NJ, Yamamoto M, et al. The myristoylation of TRIF-related adaptor molecule is essential for Toll-like receptor 4 signal transduction. Proc Natl Acad Sci U S A. 2006;103:6299-304.

44. Zhu YP, Luo SK, Sabo Y, Wang C, Tong L, Goff SP. Heme oxygenase 2 binds myristate to regulate retrovirus assembly and TLR4 signaling. Cell Host Microbe. 2017;21:220-30.

45. Timms RT, Zhang Z, Rhee DY, Harper JW, Koren I, Elledge SJ. A glycine-specific Ndegron pathway mediates the quality control of protein $\mathrm{N}$-myristoylation. Science. 2019;365:1-13.

46. Patwardhan $\mathrm{P}$, Resh MD. Myristoylation and membrane binding regulate c-Src stability and kinase activity. Mol Cell Biol. 2010;30:4094-107.

47. Belda-Palazon B, Julian J, Coego A, Wu Q, Zhang X, Batistic O, et al. ABA inhibits myristoylation and induces shuttling of the RGLG1 E3 ligase to promote nuclear degradation of PP2CA. Plant J. 2019;98:813-25.

48. Lee SC, Shaw BD. A novel interaction between N-myristoylation and the 26S proteasome during cell morphogenesis. Mol Microbiol. 2007;63:1039-53.

49. Hantschel O, Nagar B, Guettler S, Kretzschmar J, Dorey K, Kuriyan J, et al. A myristoyl/phosphotyrosine switch regulates c-Abl. Cell. 2003;112:845-57.

50. Oakhill JS, Chen ZP, Scott JW, Steel R, Castelli LA, Ling N, et al. beta-Subunit myristoylation is the gatekeeper for initiating metabolic stress sensing by AMPactivated protein kinase (AMPK). Proc Natl Acad Sci U S A. 2010;107:19237-41.

51. Wen Z, Jin K, Shen Y, Yang Z, Li Y, Wu B, et al. N-myristoyltransferase deficiency impairs activation of kinase AMPK and promotes synovial tissue inflammation. Nat Immunol. 2019;20:313-25.

52. Alonso AM, Turowski VR, Ruiz DM, Orelo BD, Moresco JJ, Yates JR, et al. Exploring protein myristoylation in Toxoplasma gondii. Exp Parasitol. 2019;203:8-18.

53. Tillo $\mathrm{SE}$, Xiong $\mathrm{WH}$, Takahashi $\mathrm{M}$, Miao $\mathrm{S}$, Andrade AL, Fortin DA, et al. Liberated PKA Catalytic subunits associate with the membrane via myristoylation to preferentially phosphorylate membrane substrates. Cell Rep. 2017;19:617-29.

54. Eustace NJ, Anderson JC, Langford CP, Trummell HQ, Hicks PH, Jarboe JS, et al. Myristoylated alanine-rich C-kinase substrate effector domain phosphorylation regulates the growth and radiation sensitization of glioblastoma. Int J Oncol. 2019;54:2039-53.

55. Rajala RV, Datla RS, Carlsen SA, Anderson DH, Qi Z, Wang JH, et al. Phosphorylation of human $\mathrm{N}$-myristoyltransferase by $\mathrm{N}$-myristoylated SRC family tyrosine kinase members. Biochem Biophys Res Commun. 2001;288:233-9.
56. Selvakumar P, Lakshmikuttyamma A, Shrivastav A, Das SB, Dimmock JR, Sharma RK. Potential role of $\mathrm{N}$-myristoyltransferase in cancer. Prog Lipid Res. 2007;46:1-36

57. Martin DD, Vilas GL, Prescher JA, Rajaiah G, Falck JR, Bertozzi CR, et al. Rapid detection, discovery, and identification of post-translationally myristoylated proteins during apoptosis using a bio-orthogonal azidomyristate analog. FASEB J. 2008;22:797-806.

58. Beauchamp E, Goenaga D, Le Bloc'h J, Catheline D, Legrand P, Rioux V. Myristic acid increases the activity of dihydroceramide Delta 4-desaturase 1 through its $\mathrm{N}$ terminal myristoylation. Biochimie. 2007;89:1553-61.

59. Takada R, Satomi $Y$, Kurata T, Ueno N, Norioka S, Kondoh H, et al. Monounsaturated fatty acid modification of Wnt protein: Its role in Wnt secretion. Dev Cell. 2006;11:791-801.

60. Landlinger C, Salzer U, Prohaska R. Myristoylation of human LanC-like Protein 2 (LANCL2) is essential for the interaction with the plasma membrane and the increase in cellular sensitivity to adriamycin. Bba-Biomembranes. 2006;1758:1759-67.

61. Raju RVS, Datla RSS, Warrington RC, Sharma R. Effects of L-histidine and its structural analogues on human N-myristoyltransferase activity and importance of EEVEH amino acid sequence for enzyme activity (vol 37, pg 14928, 1998). Biochem-Us. 1998;37:14928-36.

62. Shrivastav A, Suri SS, Mohr R, Janardhan KS, Sharma RK, Singh B. Expression and activity of $\mathrm{N}$-myristoyltransferase in lung inflammation of cattle and its role in neutrophil apoptosis. Vet Res. 2010;41:1-12.

63. Rampoldi F, Brunk F, Bonrouhi M, Federico G, Krunic D, Porubsky S, et al. Deficiency of $\mathrm{N}$-myristoylation reveals calcineurin activity as regulator of IFN-gammaproducing gamma delta T cells. J Leukoc Biol. 2017;101:1005-14.

64. Udenwobele DI, Su RC, Good SV, Ball TB, Shrivastav SV, Shrivastav A. Myristoylation: an important protein modification in the immune response. Front Immunol. 2017;8:1-16.

65. Finlay DK. N-myristoylation of AMPK controls T cell inflammatory function. Nat Immunol. 2019;20:252-4.

66. Gottlieb-Abraham E, Gutman O, Pai GM, Rubio I, Henis YI. The residue at position 5 of the N-terminal region of Src and Fyn modulates their myristoylation, palmitoylation, and membrane interactions. Mol Biol Cell. 2016;27:3926-36.

67. Alonso A, Narisawa S, Bogetz J, Tautz L, Hadzic R, Huynh H, et al. VHY, a novel myristoylated testis-restricted dual specificity protein phosphatase related to VHX. J Biol Chem. 2004;279:32586-91.

68. Bielawska A, Bielawski J, Szulc ZM, Mayroo N, Liu X, Bai A, et al. Novel analogs of D-e-MAPP and B13. Part 2: signature effects on bioactive sphingolipids. Bioorgan Med Chem. 2008;16:1032-45.

69. Faergeman NJ, Knudsen J. Role of long-chain fatty acyl-CoA esters in the regulation of metabolism and in cell signalling. Biochem J. 1997;323(Pt 1):1-12.

70. Szulc ZM, Mayroo N, Bai AP, Bielawski J, Liu X, Norris JS, et al. Novel analogs of De-MAPP and B13. Part 1: Synthesis and evaluation as potential anticancer agents. Bioorgan Med Chem. 2008;16:1015-30.

71. Kim S, Alsaidan OA, Goodwin O, Li QJ, Sulejmani E, Han Z, et al. Blocking myristoylation of Src inhibits its kinase activity and suppresses prostate cancer progression. Cancer Res. 2017;77:6950-62.

72. Bhandarkar SS, Bromberg J, Carrillo C, Selvakumar P, Sharma RK, Perry BN, et al. Tris (Dibenzylideneacetone) dipalladium, a N-myristoyltransferase-1 inhibitor, is effective against melanoma growth in vitro and in vivo. Clin Cancer Res. 2008;14:5743-8.

73. Schoepfer J, Jahnke W, Berellini G, Buonamici S, Cotesta S, Cowan-Jacob SW, et al. Discovery of asciminib (ABL001), an allosteric inhibitor of the tyrosine kinase activity of BCR-ABL1. J Med Chem. 2018;61:8120-35.

74. Radi M, Schenone S, Botta M. Allosteric inhibitors of Bcr-Abl: towards novel myristate-pocket binders. Curr Pharm Biotechnol. 2013;14:477-87.

75. Wylie AA, Schoepfer J, Jahnke W, Cowan-Jacob SW, Loo A, Furet $P$, et al. The allosteric inhibitor $A B L 001$ enables dual targeting of BCR-ABL1. Nature. 2017;543:733-7.

76. Fabbro D, Manley PW, Jahnke W, Liebetanz J, Szyttenholm A, Fendrich G, et al. Inhibitors of the Abl kinase directed at either the ATP- or myristate-binding site. Bba-Proteins Proteom. 2010;1804:454-62.

77. King MJ, Sharma RK. Differential activation of bovine brain N-myristoyltransferase(S) by a cytosolic activator. Biochem Biophys Res Commun. 1995;212:580-8.

78. Soupene E, Kuypers FA. ACBD6 protein controls acyl chain availability and specificity of the N-myristoylation modification of proteins. J Lipid Res. 2019;60:624-35.

79. Thinon E, Morales-Sanfrutos J, Mann DJ, Tate EW. N-myristoyltransferase inhibition induces ER-stress, cell cycle arrest, and apoptosis in cancer cells. Acs Chem Biol. 2016;11:2165-76. 